\title{
Korteweg-deVries-Burgers (KdVB) equation in a five component cometary plasma with kappa described electrons and ions
}

\author{
Manesh Michael $^{1} \cdot$ Neethu T. Willington ${ }^{2} \cdot$ Neethu Jayakumar $^{1} \cdot$ Sijo Sebastian $^{1}$ \\ G. Sreekala $^{1} \cdot$ Chandu Venugopal $^{1}$
}

Received: 22 January 2016/ Accepted: 4 July 2016/Published online: 13 July 2016

(c) The Author(s) 2016. This article is published with open access at Springerlink.com

\begin{abstract}
We investigate the existence of ion-acoustic shock waves in a five component cometary plasma consisting of positively and negatively charged oxygen ions, kappa described hydrogen ions, hot solar electrons, and slightly colder cometary electrons. The KdVB equation has been derived for the system, and its solution plotted for different kappa values, oxygen ion densities, as well as the temperature ratios for the ions. It is found that the amplitude of the shock wave decreases with increasing kappa values. The strength of the shock profile decreases with increasing temperatures of the positively charged oxygen ions and densities of negatively charged oxygen ions.
\end{abstract}

Keywords Cometary plasma - Ion-acoustic solitary wave Shock wave $\cdot$ KdVB equation

\section{Introduction}

Propagation of nonlinear waves in astrophysical plasmas has generated a lot of interest in the plasma community: a large number of investigations are on-going on nonlinear waves, such as solitons, shocks, double layers, and so on, which are observed in space, astrophysical, and laboratory plasmas.

Chandu Venugopal

cvgmgphys@yahoo.co.in

1 School of Pure and Applied Physics, Mahatma Gandhi University, Priyadarshini Hills, Kottayam 686 560, Kerala, India

2 Department of Physics, C. M. S. College, Kottayam 686001, Kerala, India
Most of these studies have focussed on the nonlinearity of the ion acoustic wave, which is an important wave in plasma. The first nonlinear analysis of the ion acoustic wave was by Sagdeev [1]; its first experimental observation was by Ikezi et al. [2]. Outside of the laboratory, the observations by Viking and Freja spacecrafts have identified solitary structures in the magnetosphere as density depressions.

A nonlinear and dispersive medium always supports a solitary wave. In a soliton structure, the nonlinearity is balanced by the dispersion. The Korteweg-deVries (KdV) equation, having negative nonlinear and positive dispersive effects, will give rise to rarefactive solitons. When both nonlinearity and dispersive effects are positive, the KdV equation may result in a compressive soliton. Such compressive solitons have been studied, for example, in a moving electron-positron plasma containing positively and negatively charged dust [3]. However, a medium having significant dissipative effect and dispersion supports the formation of shock waves instead of solitons which is best described by the Korteweg-deVries-Burgers (KdVB) equation. The dissipative Burger term in the nonlinear KdVB equation arises due to dissipative mechanisms, such as wave-particle interactions, turbulence, dust charge fluctuations in a dusty plasma, multiion streaming, Landau damping, anomalous viscosity, etc. [4-6]. When wave breaking due to nonlinearity is balanced by the combined effect of dispersion and dissipation, a monotonic or oscillatory dispersive shock wave is generated in a plasma [7]. If the dissipative effect is negligible the KdVB equation transforms into the $\mathrm{KdV}$ equation which permits a soliton solution. The KdVB equation has thus been extensively used to study the properties of solitons and shock waves in dusty plasmas [8-12]. 
The presence of high energy particles in the tail of a distribution causes plasmas observed in different space environments to deviate significantly from the well-known Maxwellian distribution. Using solar wind data, Vasyliunas first predicted a non-Maxwellian distribution [13]; this distribution, which later came to be known as the "kappa distribution," has been found in many magnetospheric and astrophysical environments. Ion-acoustic shock waves in plasmas consisting of superthermal electrons and positrons have been studied recently by many authors [14-20]; the effects of electrons and positrons on ion acoustic solitary waves have also been studied [21].

A cometary plasma is composed of hydrogen, and new born heavier ions and electrons with relative densities depending on their distances from the nucleus. Initially, positively charged oxygen ions were treated as the main heavier ion [22, 23]. However, the discovery of negatively charged oxygen ions [24] enables one to consider the plasma environment around a comet as a pair-ion plasma $\left(\mathrm{O}^{+}, \mathrm{O}^{-}\right)$with other ions (both lighter and heavier) constituting the other components of the plasma.

Thus, a cometary plasma is a true multi-ion plasma consisting of both lighter and heavier ions and electrons with different temperatures. We thus model our plasma as consisting of a pair-ion plasma of oxygen ions, lighter hydrogen ions, and two components of electrons with different temperatures. The lighter hydrogen ions and electrons are modeled by kappa distributions.

A very complex structure of multiple sub-shocks and interplanetary structures of comet Halley was reported by Giotto. In addition, unambiguous observations of bow shock crossings were provided by Vega-1 [25]. These shock structures are seriously affected by heavier ions due to mass loading of the solar wind and pickup driven ion instabilities [25]. And these heavy ions can be both positively and negatively charged as discussed above. In addition, in a recent study, Voelzke and Izaguirre, analyzing 886 images of comet Halley, identified 41 solitary structures [26].

The stability of ion acoustic waves in a four component dusty plasma has been investigated recently by several authors. Early studies on nonlinear wave propagation in four component dusty plasmas were by Sakanaka and Spassovska [27] and Verheest [28]. Various aspects of dust acoustic waves have been considered in the studies by several authors [29-36]. Other nonlinear electrostatic waves have been considered by Ghosh et al. [37, 38] and Dutta et al. [39].

For reasons given at the middle of this section, a reasonably accurate modeling of a cometary plasma requires at least five components. We, therefore, investigate the existence of ion-acoustic shock waves in a five component cometary plasma consisting of positively and negatively charged oxygen ions, kappa described hydrogen ions, hot solar electrons and slightly colder cometary electrons. Related studies of the effects of two types of electrons on ion-acoustic solitary waves are those by Khaled [40] and Shahmansouri et al. [41]. Similarly, the dust acoustic shock wave has also been studied in a dusty plasma with kappa distributed ions [11]. The KdVB equation is derived for this system. It is found that the amplitude of shock wave decreases with increasing kappa values. Its strength decreases with increasing temperatures of positively charged oxygen ions and densities of negatively charged oxygen ions.

\section{Basic equations}

We consider the existence of ion-acoustic shock waves in a five component plasma consisting of negatively and positively charged oxygen ions (represented, respectively, by subscripts ' 1 ' and ' 2 '), kappa described hydrogen ions, hot electrons of solar origin and colder electrons of cometary origin. At equilibrium, charge neutrality requires that

$n_{\mathrm{ce} 0}+n_{\mathrm{se} 0}+Z_{1} n_{10}=n_{\mathrm{H} 0}+Z_{2} n_{20}$.

In the above relation, $n_{\mathrm{ce} 0}$ and $n_{\mathrm{he} 0}$ represent the equilibrium densities of cometary electrons and solar electrons, respectively. In addition, $n_{10}, n_{20}$, and $n_{\mathrm{H} 0}$ are, respectively, the equilibrium densities of negatively charged oxygen $\left(\mathrm{O}^{-}\right)$ions, positively charged oxygen $\left(\mathrm{O}^{+}\right)$ions, and hydrogen ions. $\mathrm{Z}_{1}$ and $\mathrm{Z}_{2}$ denote the charge numbers of $\mathrm{O}^{-}$ and $\mathrm{O}^{+}$ions, respectively.

The kappa distribution of species ' $\mathrm{s}$ ' is given by:

$n_{\mathrm{s}}=n_{\mathrm{s} 0}\left[1+\frac{e_{\mathrm{s}} \varphi}{k_{\mathrm{B}} T_{\mathrm{s}}\left(\kappa_{s}-3 / 2\right)}\right]^{-\kappa_{\mathrm{s}}+1 / 2}$.

In (1), $\mathrm{s}=\mathrm{H}$ for hydrogen, = se for solar electrons, and $=$ ce for cometary photo-electrons. $n_{\mathrm{s}}$ denotes the density (with the subscript ' 0 ' denoting the equilibrium value), $e_{\mathrm{s}}$ is the charge, $T_{\mathrm{s}}$ is the temperature, $\kappa_{\mathrm{s}}$ is the spectral index for species 's', $k_{\mathrm{B}}$ is Boltzmann's constant, and $\varphi$ is the potential.

The dynamics of the heavier ions can be described by the following hydrodynamic equations:

$$
\begin{aligned}
& \frac{\partial n_{j}}{\partial t}+\frac{\partial\left(n_{j} v_{j}\right)}{\partial x}=0 \\
& \left(\frac{\partial}{\partial t}+v_{j} \frac{\partial}{\partial x}\right) v_{j}=\mp \frac{Z_{j} e}{m_{j}} \frac{\partial \varphi}{\partial x}-\frac{1}{m_{j} n_{j}} \frac{\partial P_{j}}{\partial x}+\mu_{j} \frac{\partial^{2} v_{j}}{\partial x^{2}},
\end{aligned}
$$

where '-' sign refers to positively charged oxygen ions (and vice versa) and $v_{j}$ and $m_{j}$, respectively, denote the fluid velocity and mass of the $\mathrm{j}$-species of ions $\left(j=\mathrm{O}^{-}\right.$, $\mathrm{O}^{+}$). In (3), the adiabatic equation of state for ions is 
$\frac{P_{j}}{P_{j 0}}=\left(\frac{n_{j}}{n_{j 0}}\right)^{\gamma}=N_{j}^{\gamma}$, where $P_{j 0}=n_{j 0} k_{\mathrm{B}} T_{j}, \gamma=(N+2) / N$ for an $N$ dimensional system, and $\mu_{j}$ is the ion kinematic viscosity. Here, we are considering a one-dimensional system, and hence, $\gamma=3$.

Poisson's equation is given by

$\frac{\partial^{2} \varphi}{\partial x^{2}}=-4 \pi e\left(n_{\mathrm{H}}+Z_{2} n_{2}-Z_{1} n_{1}-n_{\mathrm{ce}}-n_{\mathrm{se}}\right)$.

We normalize (2)-(4) using the parameters of $\mathrm{O}^{-}$ions according to $\varphi=\frac{e \phi}{k_{\mathrm{B}} T_{1}}, V_{j}=\frac{v_{j}}{c_{\mathrm{s}}}$, and $\tau=\frac{t}{\omega_{\mathrm{pl}}^{-1}}$, where $c_{\mathrm{S}}=$ $\left(\frac{Z_{1} k_{\mathrm{B}} T_{1}}{m_{1}}\right)^{1 / 2}$ and $\omega_{\mathrm{p} 1}=\left(\frac{4 \pi Z_{1}^{2} e^{2} n_{10}}{m_{1}}\right)^{1 / 2}$. The variable $x$ is normalized using $\lambda_{\mathrm{D} 1}=\left(\frac{Z_{1} k_{\mathrm{B}} T_{1}}{4 \pi Z_{1}^{2} e^{2} n_{10}}\right)^{1 / 2}$, while $N_{j}=\frac{n_{j}}{n_{j 0}}$.

Thus, Eqs. (2)-(4) can be rewritten as:

$\frac{\partial N_{1}}{\partial \tau}+\frac{\partial\left(N_{1} V_{1}\right)}{\partial x}=0$,

$\frac{\partial N_{2}}{\partial \tau}+\frac{\partial\left(N_{2} V_{2}\right)}{\partial x}=0$

$\frac{\partial V_{1}}{\partial \tau}+V_{1} \frac{\partial V_{1}}{\partial x}=\frac{\partial \phi}{\partial x}-\frac{3 N_{1}}{Z_{1}} \frac{\partial N_{1}}{\partial x}+\rho_{1} \frac{\partial^{2} V_{1}}{\partial x^{2}}$,

$\frac{\partial V_{2}}{\partial \tau}+V_{2} \frac{\partial V_{2}}{\partial x}=\frac{-Z_{2} m}{Z_{1}} \frac{\partial \phi}{\partial x}-\frac{3 m \beta N_{2}}{Z_{1}} \frac{\partial N_{2}}{\partial x}+\rho_{2} \frac{\partial^{2} V_{2}}{\partial x^{2}}$,

where $m=\frac{m_{1}}{m_{2}}, \beta=\frac{T_{2}}{T_{1}}, \rho_{1}=\frac{\mu_{1}}{\omega_{\mathrm{p} 1} \lambda_{\mathrm{D}}^{2}}$, and $\rho_{2}=\frac{\mu_{2}}{\omega_{\mathrm{p} 1} \lambda_{\mathrm{D}}^{2}}$.

$\rho_{1}$ and $\rho_{2}$ now represent the normalized kinematic viscosities of the pair ions.

The normalized Poisson's equation after substitution of (1) is:

$$
\begin{aligned}
\frac{\partial^{2} \phi}{\partial x^{2}}= & N_{1}-N_{2}\left(1+\mu_{\mathrm{ce}}+\mu_{\mathrm{se}}-\mu_{\mathrm{H}}\right) \\
& +\mu_{\mathrm{ce}}\left(1-\frac{\phi}{\sigma_{c e}\left(\kappa_{c e}-3 / 2\right)}\right)^{-\left(\kappa_{\mathrm{ce}}-1 / 2\right)} \\
& +\mu_{\mathrm{se}}\left(1-\frac{\phi}{\sigma_{\mathrm{se}}\left(\kappa_{\mathrm{se}}-3 / 2\right)}\right)^{-\left(\kappa_{\mathrm{se}}-1 / 2\right)} \\
& -\mu_{\mathrm{H}}\left(1+\frac{\phi}{\sigma_{\mathrm{H}}\left(\kappa_{\mathrm{H}}-3 / 2\right)}\right)^{-\left(\kappa_{\mathrm{H}}-1 / 2\right)}
\end{aligned}
$$

where $\quad \mu_{\mathrm{ce}}=\frac{n_{\mathrm{ce} 0}}{Z_{1} n_{10}}, \quad \mu_{\mathrm{se}}=\frac{n_{\mathrm{se} 0}}{Z_{1} n_{10}}, \quad \mu_{\mathrm{H}}=\frac{n_{\mathrm{H} 0}}{Z_{1} n_{10}}, \quad \sigma_{\mathrm{ce}}=\frac{T_{\mathrm{ce}}}{T_{1}}$, $\sigma_{\mathrm{se}}=\frac{T_{\mathrm{se}}}{T_{1}}$, and $\sigma_{\mathrm{H}}=\frac{T_{\mathrm{H}}}{T_{1}}$.

\section{Derivation of KdVB equation}

We use the reductive perturbation method to derive the KdVB equation from (5) to (9) by introducing the transformations

$$
\xi=\varepsilon^{1 / 2}(x-\lambda t), \quad \tau=\varepsilon^{3 / 2} t, \quad \rho_{j}=\varepsilon^{1 / 2} \rho_{j 0}
$$

where $\varepsilon$ is a smallness parameter and $\lambda$ is the wave phase speed.

To apply the reductive perturbation technique, the various parameters are expanded as:

$N_{1,2}=1+\varepsilon N_{1,2}^{(1)}+\varepsilon^{2} N_{1,2}^{(2)}+\cdots$

$V_{(1,2)}=\varepsilon V_{(1,2)}^{(1)}+\varepsilon^{2} V_{(1,2)}^{(2)}+\cdots$

$\phi=\varepsilon \phi^{(1)}+\varepsilon^{2} \phi^{(2)}+\cdots$

We substitute (10)-(12) in (5)-(9) and equate the coefficients of different powers of $\varepsilon$. From the coefficients of order $\varepsilon^{3 / 2}$, we get the first-order terms as:

$N_{1}^{1}=\frac{\phi^{1}}{\left(\frac{3}{Z_{1}}-\lambda^{2}\right)}$

and

$N_{2}^{1}=\frac{\left(Z_{2} / Z_{1}\right) m \phi^{1}}{\left(\lambda^{2}-\frac{3 m \beta}{Z_{1}}\right)}$.

Expressions for $V_{1}^{1}$ and $V_{2}^{1}$ can be obtained by multiplying (13) and (14) by $\lambda$.

In addition, the linear dispersion relation is:

$\lambda^{2}=\frac{S \pm \sqrt{S^{2}-12 m Z_{1}^{2} T\left[3 m \beta T+m Z_{2}\left(1+\mu_{c e}+\mu_{s e}-\mu_{H}\right)+m \beta Z_{1}\right]}}{2 T Z_{1}^{2}}$,

where

$S=Z_{1}^{2}+m Z_{1} Z_{2}\left(1+\mu_{c e}+\mu_{s e}-\mu_{H}\right)+3 Z_{1} T(1+m \beta)$,

and

$T=\frac{\mu_{\mathrm{ce}}\left(\kappa_{\mathrm{ce}}-1 / 2\right)}{\left(\kappa_{\mathrm{ce}}-3 / 2\right) \sigma_{\mathrm{ce}}}+\frac{\mu_{\mathrm{se}}\left(\kappa_{\mathrm{se}}-1 / 2\right)}{\left(\kappa_{\mathrm{se}}-3 / 2\right) \sigma_{\mathrm{se}}}+\frac{\mu_{\mathrm{H}}\left(\kappa_{\mathrm{H}}-1 / 2\right)}{\left(\kappa_{\mathrm{H}}-3 / 2\right) \sigma_{\mathrm{H}}}$.

Equating the coefficients of $\varepsilon^{5 / 2}$ in (5) and (6), we get

$\frac{\partial N_{i}^{1}}{\partial \tau}-\lambda \frac{\partial N_{i}^{2}}{\partial \xi}+\frac{\partial V_{i}^{2}}{\partial \xi}+\frac{\partial\left(N_{i}^{1} V_{i}^{1}\right)}{\partial \xi}=0 \quad i=1,2$.

And equating the coefficient of order $\varepsilon^{5 / 2}$ in (7) and (8) results in:

$$
\begin{aligned}
\frac{\partial V_{1}^{1}}{\partial \tau}-\lambda \frac{\partial V_{1}^{2}}{\partial \xi}+V_{1}^{1} \frac{\partial V_{1}^{1}}{\partial \xi}= & \frac{\partial \phi^{2}}{\partial \xi}-\frac{3 N_{1}^{1}}{Z_{1}} \frac{\partial N_{1}^{1}}{\partial \xi}-\frac{3}{Z_{1}} \frac{\partial N_{1}^{2}}{\partial \xi} \\
& +\rho_{10} \frac{\partial^{2} V_{1}^{1}}{\partial \xi^{2}},
\end{aligned}
$$

$$
\begin{aligned}
\frac{\partial V_{2}^{1}}{\partial \tau}-\lambda \frac{\partial V_{2}^{2}}{\partial \xi}+V_{2}^{1} \frac{\partial V_{2}^{1}}{\partial \xi}= & -\frac{Z_{2} m}{Z_{1}} \frac{\partial \phi^{2}}{\partial \xi}-\frac{3 m \beta N_{2}^{1}}{Z_{1}} \frac{\partial N_{2}^{1}}{\partial \xi} \\
& -\frac{3 m \beta}{Z_{1}} \frac{\partial N_{2}^{2}}{\partial \xi}+\rho_{20} \frac{\partial^{2} V_{2}^{1}}{\partial \xi^{2}} .
\end{aligned}
$$


Finally, equating the coefficients of terms of order $\varepsilon^{2}$ from Poisson's equation (9) gives:

$$
\begin{aligned}
\frac{\partial^{2} \phi^{1}}{\partial \xi^{2}}= & N_{1}^{2}-N_{2}^{2}\left(1+\mu_{\mathrm{ce}}+\mu_{\mathrm{se}}-\mu_{\mathrm{H}}\right)+T \phi^{2} \\
& +\frac{\mu_{\mathrm{ce}}}{2} \frac{\left(\kappa_{\mathrm{ce}}^{2}-1 / 4\right)}{\left(\kappa_{\mathrm{ce}}-3 / 2\right)^{2} \sigma_{\mathrm{ce}}^{2}}\left(\phi^{1}\right)^{2} \\
& +\frac{\mu_{\mathrm{se}}}{2} \frac{\left(\kappa_{\mathrm{se}}^{2}-1 / 4\right)}{\left(\kappa_{\mathrm{se}}-3 / 2\right)^{2} \sigma_{\mathrm{se}}^{2}}\left(\phi^{1}\right)^{2}-\frac{\mu_{\mathrm{H}}}{2} \frac{\left(\kappa_{\mathrm{H}}^{2}-1 / 4\right)}{\left(\kappa_{\mathrm{H}}-3 / 2\right)^{2} \sigma_{\mathrm{H}}^{2}}\left(\phi^{1}\right)^{2} .
\end{aligned}
$$

Substituting the values from (13) and (14) into (18) to (21) and eliminating the second-order terms, we obtain the $\mathrm{KdVB}$ equation as:

$A \frac{\partial \phi^{1}}{\partial \tau}+\phi^{1} \frac{\partial \phi^{1}}{\partial \xi}+B \frac{\partial^{3} \phi^{1}}{\partial \xi^{3}}-C \frac{\partial^{2} \phi^{1}}{\partial \xi^{2}}=0$.

In (22), the coefficients $A, B$, and $C$ are given by: dissipation, a convenient method to solve it is "the tanh method" [43, 44]. Using the above transformation, (22) can be written as:

$-A V \frac{\partial \phi^{1}}{\partial \chi}+\phi^{1} \frac{\partial \phi^{1}}{\partial \chi}+B \frac{\partial^{3} \phi^{1}}{\partial \chi^{3}}-C \frac{\partial^{2} \phi^{1}}{\partial \chi^{2}}=0$

Again using the transformation $\alpha=\tanh \chi$ and assuming a series solution of the form $\phi^{1}(\alpha)=\sum_{i=0}^{n} a_{i} \alpha^{i}$, we arrive at the solution of (22) as:

$$
\begin{aligned}
\phi^{1}= & A V+8 B k^{2}+\frac{C^{2}}{25 B}-12 k^{2} \tanh ^{2}[k(\xi-V \tau)] \\
& -\frac{12}{5} k C[1-\tanh [k(\xi-V \tau)]] .
\end{aligned}
$$

The speed of comoving frame is related to the coefficients $A, B$, and $C$ as $V=\frac{\left(100 B^{2} k^{2}-C^{2}+60 k B C\right)}{25 A B}$ and $k=\frac{ \pm C}{10 B}$ which can be obtained using the boundary conditions.

$A=\frac{-2 Z_{1}^{2} \lambda\left(Z_{1} \lambda^{2}-3\right)\left(Z_{1} \lambda^{2}-3 m \beta\right)\left[\left(Z_{1} \lambda^{2}-3 m \beta\right)^{2}+m\left(\frac{Z_{2}}{Z_{1}}\right)\left(1+\mu_{\mathrm{ce}}+\mu_{\mathrm{se}}-\mu_{\mathrm{H}}\right)\left(Z_{1} \lambda^{2}-3\right)^{2}\right]}{D}$

$B=\frac{-\left(Z_{1} \lambda^{2}-3\right)^{3}\left(Z_{1} \lambda^{2}-3 m \beta\right)^{3}}{D}$

$C=\frac{-Z_{1}^{2} \lambda\left(Z_{1} \lambda^{2}-3\right)\left(Z_{1} \lambda^{2}-3 m \beta\right)\left[\rho_{10}\left(Z_{1} \lambda^{2}-3 m \beta\right)^{2}-m \rho_{20}\left(\frac{Z_{2}}{Z_{1}}\right)\left(1+\mu_{\mathrm{ce}}+\mu_{\mathrm{se}}-\mu_{\mathrm{H}}\right)\left(Z_{1} \lambda^{2}-3\right)^{2}\right]}{D}$

where

$L=\frac{\mu_{\mathrm{ce}}\left(\kappa_{\mathrm{ce}}^{2}-1 / 4\right)}{\left(\kappa_{\mathrm{ce}}-3 / 2\right)^{2} \sigma_{\mathrm{ce}}^{2}}+\frac{\mu_{\mathrm{se}}\left(\kappa_{\mathrm{se}}^{2}-1 / 4\right)}{\left(\kappa_{\mathrm{se}}-3 / 2\right)^{2} \sigma_{\mathrm{se}}^{2}}-\frac{\mu_{\mathrm{H}}\left(\kappa_{\mathrm{H}}^{2}-1 / 4\right)}{\left(\kappa_{\mathrm{H}}-3 / 2\right)^{2} \sigma_{\mathrm{H}}^{2}}$

and

$$
\begin{aligned}
D= & L\left(Z_{1} \lambda^{2}-3\right)^{3}\left(Z_{1} \lambda^{2}-3 m \beta\right)^{3}+3 Z_{1}^{2}\left(1+Z_{1} \lambda^{2}\right) \\
& \times\left(Z_{1} \lambda^{2}-3 m \beta\right)^{3}-3 m^{2} Z_{2}^{2}\left(1+\mu_{\mathrm{ce}}+\mu_{\mathrm{se}}-\mu_{\mathrm{H}}\right) \\
& \times\left(Z_{1} \lambda^{2}-3\right)\left(Z_{1} \lambda^{2}+m \beta\right) .
\end{aligned}
$$

\section{Solution of KdVB equation}

To find the solution of (22), we use the transformed coordinate $\chi=(\xi-V \tau)$ of the comoving frame with speed $V$ and use the boundary conditions: $\phi^{1} \rightarrow 0$ and $\frac{\partial \phi^{1}}{\partial \chi}, \frac{\partial^{2} \phi^{1}}{\partial \chi^{2}}$, $\frac{\partial^{3} \phi^{1}}{\partial \chi^{3}} \rightarrow 0$ as $\chi \rightarrow \infty$ for a localized solution [42].

When the partial differential equation of a system is formed by the combined effect of dispersion and

\section{Results}

Solution (25) is applicable to any astrophysical plasma. However, in this study, we concentrate on parameters relevant to comet Halley: the observed value of the density of hydrogen ions was $n_{\mathrm{H}}=4.95 \mathrm{~cm}^{-3}$; their temperature was

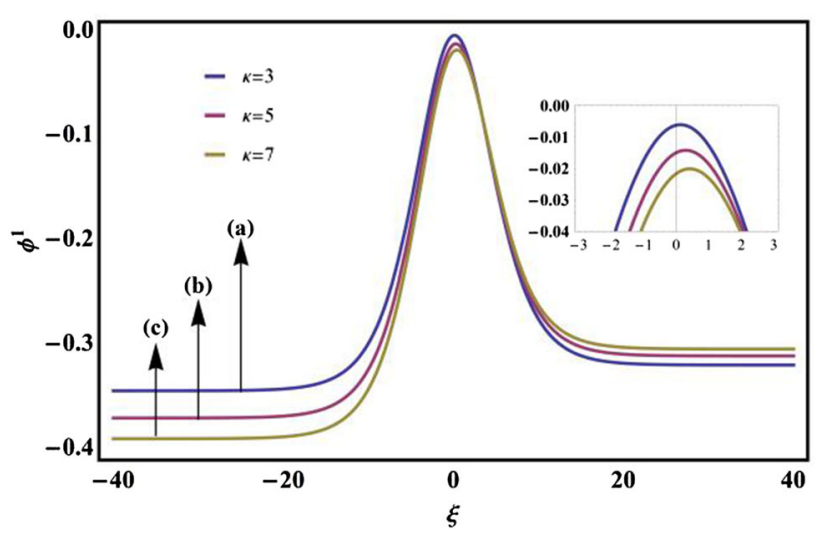

Fig. $1 \phi^{1}$ vs $\chi$ as a function of kappa indices 


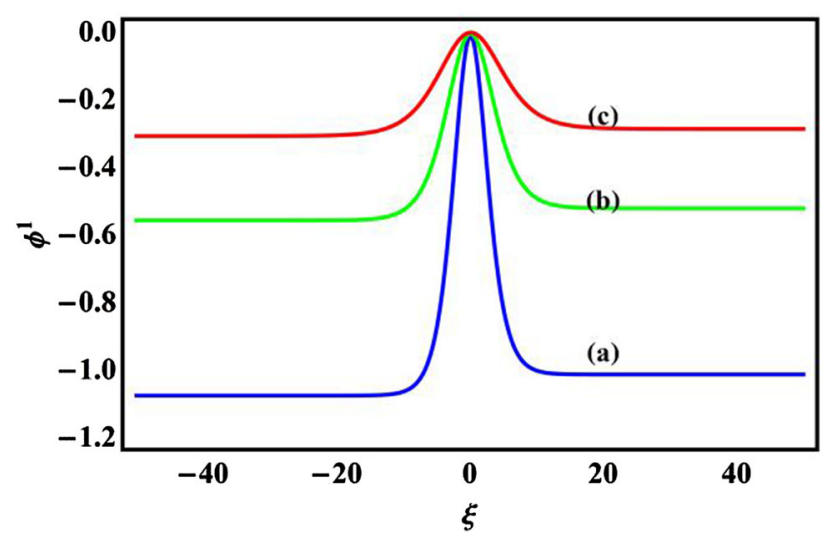

Fig. $2 \phi^{1}$ vs $\chi$ as a function of temperature ratio $\beta$

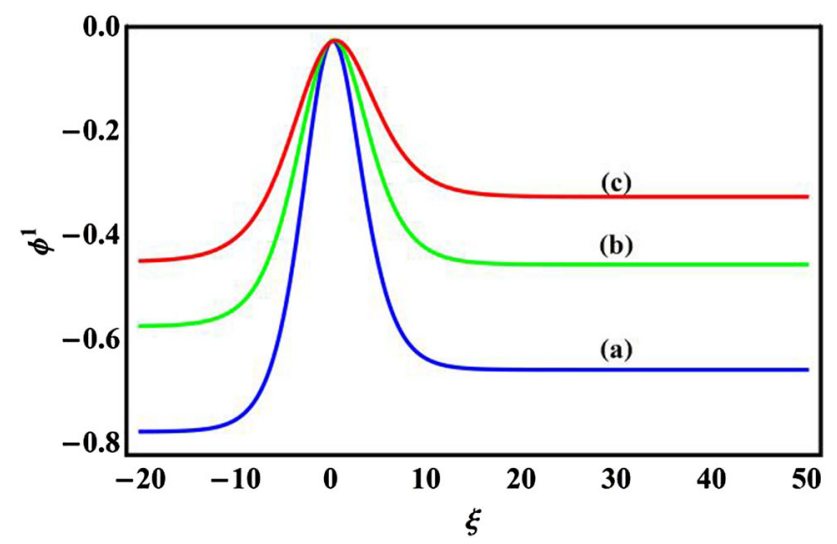

Fig. $3 \phi^{1}$ vs $\chi$ as a function of $\mathrm{O}^{-}$density

$T_{\mathrm{H}}=8 \times 10^{4} \mathrm{~K}$. The temperature of the solar (or hot) electrons was $T_{\mathrm{se}}=2 \times 10^{5} \mathrm{~K}$ [45]. The temperature of the second component of electrons, namely photoelectrons was set at $T_{\text {ce }}=2 \times 10^{4} \mathrm{~K}$. Negatively charged oxygen ions with an energy $\sim 1 \mathrm{eV}$ and densities $\leq 1 \mathrm{~cm}^{-3}$ was identified by Chaizy et al. [24]. We thus set the densities of positively charged oxygen ions at $n_{20}=0.5 \mathrm{~cm}^{-3}$ and that of negatively charged oxygen ions at $n_{10}=0.05 \mathrm{~cm}^{-3}$ $[24,45]$.

Figure 1 is a plot of the solution (25) of the KdVB Eq. (22), and shows the variation of the potential $\varphi^{1}$ versus $\chi$ as a function of the spectral indices; the parameters for the figure are $n_{10}=0.05 \mathrm{~cm}^{-3}, n_{20}=0.5 \mathrm{~cm}^{-3}, n_{\mathrm{H}}=$ $4.95 \mathrm{~cm}^{-3}, \quad T_{\mathrm{ce}}=2 \times 10^{4} \mathrm{~K}, \quad T_{\mathrm{se}}=2 \times 10^{5} \mathrm{~K}, \quad T_{1}=T_{2}=$ $1.16 \times 10^{4} \mathrm{~K}$, and $Z_{1}=Z_{2}=1$. Curve (a) is for the spectral index $\kappa=3$, curve (b) is for $\kappa=5$, and curve (c) is for $\kappa=7$. It is clear from the figure that the amplitude of shock wave increases with a decrease of kappa indices.

Figure 2 is a plot of the potential $\varphi^{1}$ versus $\chi$ as a function of temperature ratio $\left(\beta=\frac{T_{2}}{T_{1}}\right)$ of the pair ions. We keep the spectral indices at $\kappa_{\mathrm{ce}}=\kappa_{\mathrm{se}}=\kappa_{\mathrm{H}}=3$; the other

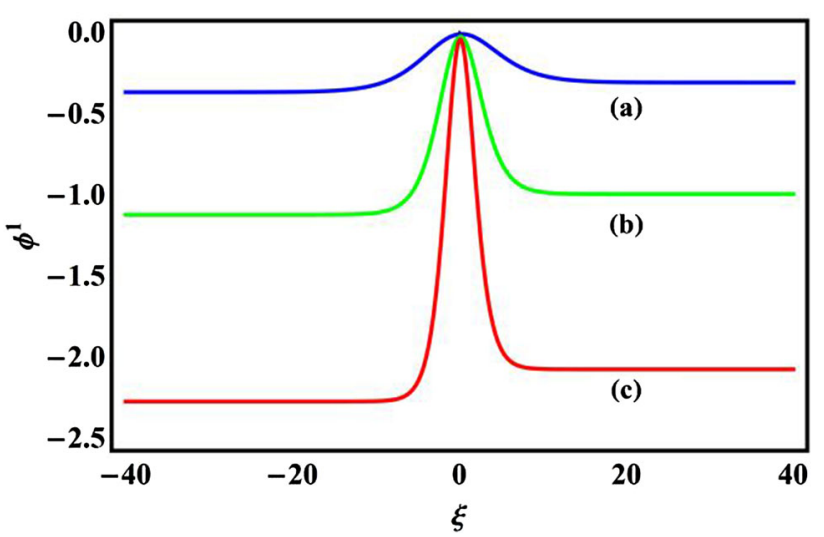

Fig. $4 \phi^{1}$ vs $\chi$ as a function of $\mathrm{O}^{+}$density

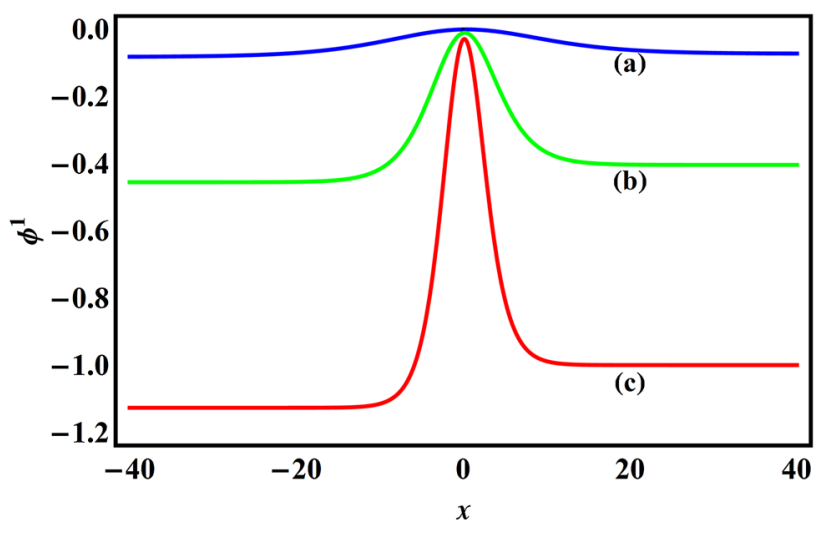

Fig. $5 \phi^{1}$ vs $\chi$ as a function of kinematic viscosity of $\mathrm{O}^{+}$ions

parameters are the same as in Fig. 1. Curve (a) is for $\beta=2$, curve (b) is for $\beta=2.2$, and curve (c) is for $\beta=2.4$. We find that the strength of shock profile decreases with an increase of the temperature of positively charged oxygen ions.

Figure 3 is again a plot of the potential $\varphi^{1}$ versus $\chi$ as a function of the negative oxygen ion densities $n_{10}$. The parameters used in this case are $n_{20}=0.5 \mathrm{~cm}^{-3}, n_{\mathrm{H}}=$ $4.95 \mathrm{~cm}^{-3}, \quad T_{\mathrm{ce}}=2 \times 10^{4} \mathrm{~K}, \quad T_{\mathrm{se}}=2 \times 10^{5} \mathrm{~K}, \quad T_{1}=T_{2}=$ $1.16 \times 10^{4} K, Z_{1}=Z_{2}=1$, and $\kappa_{\mathrm{ce}}=\kappa_{\mathrm{se}}=\kappa_{\mathrm{H}}=5$. Curve (a) is for $n_{10}=0.06 \mathrm{~cm}^{-3}$, curve (b) is for $n_{10}=0.07 \mathrm{~cm}^{-3}$, and curve (c) is for $n_{10}=0.08 \mathrm{~cm}^{-3}$. We find that the strength of shock wave decreases with an increase of the negatively charged oxygen ion densities.

Figure 4 shows the variation of the amplitude of shock wave as a function of the positively charged oxygen ion densities. The parameters chosen are the same as in Fig. 3. Here, the negatively charged oxygen ion density is $n_{10}=0.05 \mathrm{~cm}^{-3}$. Curve (a) is for $n_{20}=0.3 \mathrm{~cm}^{-3}$, curve (b) is for $n_{20}=0.5 \mathrm{~cm}^{-3}$, and curve (c) is for $n_{20}=0.7 \mathrm{~cm}^{-3}$. We find that increasing positively charged 


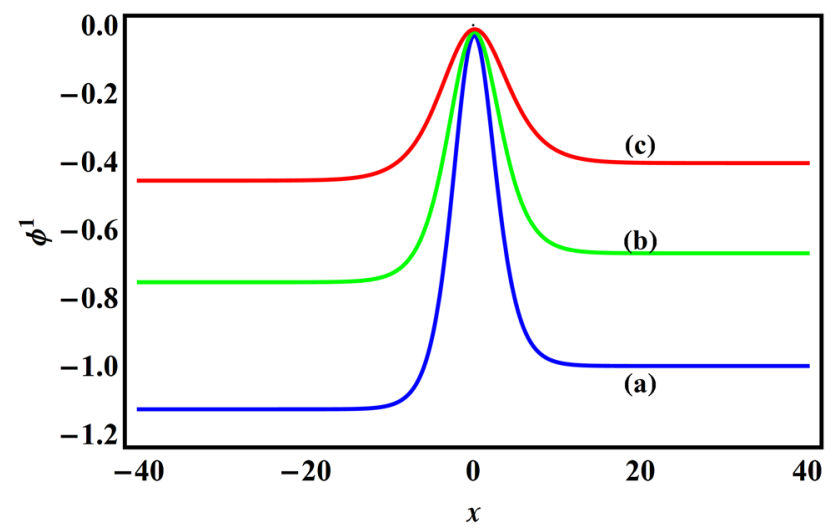

Fig. $6 \phi^{1}$ vs $\chi$ as a function of kinematic viscosity of $\mathrm{O}^{-}$ions

oxygen ion density will increase the strength of the shock wave profile.

Figure 5 is a plot of the solution of the KdVB Eq. (22) and shows the variation of the potential $\varphi^{1}$ versus $\chi$ as a function of the normalized kinematic viscosity of positively charged oxygen ions $\left(\rho_{2}\right)$; the parameters for the figure are $n_{10}=0.05 \mathrm{~cm}^{-3}, n_{20}=0.5 \mathrm{~cm}^{-3}, n_{\mathrm{H}}=4.95 \mathrm{~cm}^{-3}, T_{\text {ce }}=$ $2 \times 10^{4} \mathrm{~K}, T_{\mathrm{se}}=2 \times 10^{5} \mathrm{~K}, T_{1}=T_{2}=1.16 \times 10^{4} \mathrm{~K}$, and $Z_{1}=Z_{2}=1$. Curve (a) is for the kinematic viscosity $\rho_{2}=0.25$, curve (b) is for $\rho_{2}=0.5$, and curve (c) is for $\rho_{2}=0.75$. It is clear from the figure that the strength of shock wave increases with an increase in kinematic viscosity of positively charged oxygen ions.

Figure 6 shows the variation of the potential $\varphi^{1}$ versus $\chi$ as a function of the normalized kinematic viscosity of negatively charged oxygen ions $\left(\rho_{1}\right)$. The parameters used in this case are the same as in Fig. 5. Curve (a) is for the kinematic viscosity $\rho_{1}=0.1$, curve (b) is for $\rho_{1}=0.3$, and curve (c) is for $\rho_{1}=0.5$. We find that the strength of shock wave decreases with an increase in the kinematic viscosity of negatively charged oxygen ions.

In a study of electron acoustic solitary and shock waves in dissipative space plasmas with superthermal, hot electrons, Han et al. [46] found that the amplitude of the shock wave decreased with increasing spectral index $\kappa$. They also found that the strength of the shock wave increased with increasing ratios of the cold to hot electron densities and electron kinematic viscosities. In another study on drift ionacoustic shocks in an inhomogeneous 2-D quantum plasma, Masood et al. [47] found that the strength of the shock waves increased with increasing densities of ions.

The results of Fig. 1, which are in agreement with that of Han et al. [46], can be considered as extending their result to a multi-component plasma as we now have three components being described by kappa distributions. Similarly, the conclusions of Fig. 4 where the strength of the shock wave increases with increasing positively charged oxygen ion density is in agreement with the result of Masood et al. [47].

Finally, the other observation of Han et al. [46] that the strength of shock wave increased with increasing electron kinematic viscosity was interpreted as being due to increasing dissipation among the constituents. Increasing the density of positively charged oxygen ions would lead to an increase in the electron density; conversely, an increase in the negatively charged oxygen ion densities would lead to a decrease in the electron densities. The results of Figs. 5 and 6 are consistent with this observation of Han et al. [46].

More recently, Sabetkar and Dorranian [48] investigated the characteristics of dust acoustic solitary waves (DASWs) in a plasma composed of two populations of ions, electrons and negatively charged dust. The ions and electrons were modeled by three-dimensional non-extensive and kappa distributions, respectively. They derived the Zakharov-Kuznetsov (ZK) and modified Zakharov-Kuznetsov (mZK) equations to describe dust acoustic waves in the system. Both positive and negative polarity solutions were found to exist. They found that the amplitude of the $\mathrm{ZK}$ solitons decreased with increasing $\kappa$ values, which is the conclusion from Fig. 1. In addition, the amplitude of these solitons increased with an increase in the temperature ratio of the cold to hot ions, similar to the conclusion from Fig. 2.

In yet another study, the same authors [49] studied the "fast" and "slow" dust acoustic waves in a plasma composed of Maxwellian electrons, kappa distributed positive ions, kappa-Schamel distributed negative ions and negatively charged dust particles. The 1 and 3D SchamelKorteweg-deVries (S-KdV) equation that they derived admitted only compressive solitons. A point of convergence with their conclusion is the decrease in the amplitude of the soliton with the temperature ratio $\beta_{\mathrm{p}}\left(=\frac{T_{\mathrm{p}}}{T_{\mathrm{n}}}\right)$. Further comparisons are not possible because of the different distributions used and also the different equations studied.

\section{Conclusion}

We have, in this paper, studied ion-acoustic shock waves in a five component plasma of positively and negatively charged oxygen ions, lighter hydrogen ions, and hot and cold electrons by deriving the KdVB equation. The impact of spectral indices kappa, temperature of positively charged oxygen ions, and density of oxygen ions $\left(\mathrm{O}^{+}, \mathrm{O}^{-}\right)$on the shock wave amplitude is studied. We find that in a cometary plasma with the above components, the nonlinear wave is in a transition state from shock to soliton [20]. A reduction in the shock wave amplitude is seen with increasing spectral indices and positively charged oxygen 
ion density. The strength of the shock profile also decreases with increasing positively charged oxygen ion temperatures and negatively charged oxygen ion densities. These results can be expected to contribute to an understanding of shocks in comets as we have two heavy ion components and heavy ions were surmised to affect shocks in cometary plasmas [25].

Acknowledgments The authors thank the referees for their valuable comments. Financial assistance from Kerala State Council for Science, Technology and Environment, Thiruvananthapuram, Kerala, India (JRFs for MM and SG) and the University Grants Commission $(\mathrm{EF})$ is gratefully acknowledged.

Open Access This article is distributed under the terms of the Creative Commons Attribution 4.0 International License (http://crea tivecommons.org/licenses/by/4.0/), which permits unrestricted use, distribution, and reproduction in any medium, provided you give appropriate credit to the original author(s) and the source, provide a link to the Creative Commons license, and indicate if changes were made.

\section{References}

1. Sagdeev, R.Z., Leontovich, M.A.: Cooperative phenomena and shock waves in collisionless plasmas. Rev. Plasma Phys. 4, 23 (1966)

2. Ikezi, H., Taylor, R., Baker, D.: Formation and interaction of ionacoustic solitions. Phys. Rev. Lett. 25(1), 11 (1970)

3. Malik, R., Malik, H.K.: Compressive solitons in a moving e-p plasma under the effect of dust grains and an external magnetic field. J. Theor. Appl. Phys. 7, 65 (2013)

4. Mamun, A.A., Shukla, P.K.: Cylindrical and spherical dust ionacoustic solitary waves. Phys. Plasmas 9(4), 1468 (2002)

5. Xue, J.K.: Cylindrical and spherical dust-ion acoustic shock waves. Phys. Plasmas 10, 4893 (2003)

6. Sahu, B., Roychoudhury, R.: Quantum ion acoustic shock waves in planar and nonplanar geometry. Phys. Plasmas 14 (7), 2310 (2007)

7. Shukla, P.K., Mamun, A.A.: Solitons, shocks and vortices in dusty plasmas. New J. Phys. 5(1), 17 (2003)

8. Shukla, P.K., Mamun, A.A.: Dust-acoustic shocks in a strongly coupled dusty plasma. IEEE Tr. PS. 29, 221 (2001)

9. Mamun, A.A., Eliasson, B., Shukla, P.K.: Dust-acoustic solitary and shock waves in a strongly coupled liquid state dusty plasma with a vortex-like ion distribution. Phys. Lett. A 332, 412 (2004)

10. Ghosh, S., Gupta, M.R.: Charging-delay effect on longitudinal dust acoustic shock wave in strongly coupled dusty plasma. Phys. Plasmas 12, 092306 (2005)

11. Pakzad, H.R.: Dust acoustic shock waves in strongly coupled dusty plasmas with kappa-distributed ions. Ind. J. Phys. 86, 743 (2012)

12. El-Hanbaly, A.M., El-Shewy, E.K., Sallah, M., Darweesh, H.F.: Linear and nonlinear analysis of dust acoustic waves in dissipative space dusty plasmas with trapped ions. J. Theor. Appl. Phys. 9, 167 (2015)

13. Vasyliunas, V.M.: Low-energy electrons on the day side of the magnetosphere. J. Geophys. Res. 73(23), 7519 (1968)

14. Masood, W., Mahmood, S., Imtiaz, N.: Electrostatic shocks and solitons in pair-ion plasmas in a two-dimensional geometry. Phys. Plasmas 16, 2306 (2009)
15. Masood, W., Rizvi, H.: Two dimensional nonplanar evolution of electrostatic shock waves in pair-ion plasmas. Phys. Plasmas 19(12), 119 (2012)

16. Samanta, U.K., Chatterjee, P., Mej, M.: Soliton and shocks in pair ion plasma in presence of superthermal electrons. Astrophys. Space Sci. 345(2), 291 (2013)

17. Sultana, S., Kourakis, I., Saini, N.S., Hellberg, M.A.: Oblique electrostatic excitations in a magnetized plasma in the presence of excess superthermal electrons. Phys. Plasmas 17, 032310 (2010)

18. Kourakis, I., Sultana, S., Hellberg, M.A.: Dynamical characteristics of solitary waves, shocks and envelope modes in kappadistributed non-thermal plasmas: an overview. Plasma Phys. Control. Fusion 54(12), 124001 (2012)

19. Sultana, S., Kourakis, I.: Electron-scale electrostatic solitary waves and shocks: the role of superthermal electrons. Eur. Phys. J. D.66, 1 (2012)

20. Saeed, R., Shah, A.: Nonlinear Korteweg-de Vries-Burger equation for ion acoustic shock waves in a weakly relativistic electron-positron-ion plasma with thermal ions. Phys. Plasmas 17, 032308 (2010)

21. Ghosh, B., Banerjee, S.: Effect of nonthermal electrons and positrons on ion-acoustic solitary waves in a plasma with warm drifting ions. Ind. J. Phys. 89, 1307 (2015)

22. Ipavich, F.M., Galvin, A.B., Gloeckler, G., Hovestadt, D., Klecker, B., Scholer, M.: Comet Giacobini-Zinner: in situ observations of energetic heavy ions. Science 232, 366 (1986)

23. Coplan, M.A., Ogilvie, K.W., A’Hearn, M.F., Bochsler, P., Geiss, J.: Ion composition and upstream solar wind observations at comet Giacobini-Zinner. J. Geophys. Res. 92, 39 (1987)

24. Chaizy, P., Reme, H., Sauvaud, J.A., d'Uston, C., Lin, RP., Larson, D.E., Mitchell, D.L., Zwickl, R.D., Baker, D.N., Bame, S.J., Feldman, W.C., Fuselier, S.A., Huebner, W.F., McComas, D.J., Young, D.T.: Negative ions in the coma of comet Halley. Nature. 349, 393 (1991)

25. Coates, A.J.: Heavy ion effects on cometary shocks. Adv. Space Res. 15, 403 (1995)

26. Voelzke, M.R., Izaguirre, L.S.: Morphological analysis of the tail structures of comet P/Halley 1910 II. Planet. Space Sci. 65(1), 104 (2012)

27. Sakanaka, P.H., Spassovska, I.: Study of nonlinear phenomena in four-component dusty plasma with charge fluctuation. Phys. Scr. T131, 014040 (2008)

28. Verheest, F., Hellberg, M.A., Kourakis, I.: Acoustic solitary waves in dusty and/or multi-ion plasmas with cold, adiabatic, and hot constituents. Phys. Plasmas 15, 112309 (2008)

29. Dorranian, D., Sabetkar, A.: Dust acoustic solitary waves in a dusty plasma with two kinds of nonthermal ions at different temperatures. Phys. Plasmas 19, 013702 (2012)

30. Shahmohammadi, N., Dorranian, D.: Effect of dust charge fluctuation on multidimensional instability of dust-acoustic solitary waves in a magnetized dusty plasma with nonthermal ions. Phys. Plasmas 22, 103707 (2015)

31. Sharif Moghadam., S, Dorranian., D.: Effect of size distribution on the dust acoustic solitary waves in dusty plasma with two kinds of nonthermal ions. Adv. Mater. Sci. Eng (2013)

32. Shahmoradi, N., Dorranian, D.: Effects of variable dust size, charge and mass on the characteristics of dust acoustic solitary waves in a magnetized dusty plasma. Phys. Scr. 89, 065602 (2014)

33. El-Taibany, W.F.: Nonlinear dust acoustic waves in inhomogeneous four-component dusty plasma with opposite charge polarity dust grains. Phys. Plasmas 20, 093701 (2013)

34. Tomar, R., Malik, H.K., Dahiya, R.P.: Reflection of ion acoustic solitary waves in a dusty plasma with variable charge dust. J. Theor. Appl. Phys. 8, 126 (2014) 
35. Tomar, R., Bhatnagar, A., Malik, H.K., Dahiya, R.P.: Evolution of solitons and their reflection and transmission in a plasma having negatively charged dust grains. J. Theor. Appl. Phys. 8, 138 (2014)

36. Sabetkar, A., Dorranian, D.: Effect of obliqueness and external magnetic field on the characteristics of dust acoustic solitary waves in dusty plasma with two-temperature nonthermal ions. J. Theor. Appl. Phys. 9, 141 (2015)

37. Ghosh, S., Sarkar, S., Khan, M., Gupta, M.R.: Nonlinear properties of small amplitude dust ion acoustic solitary waves. Phys. Plasmas 7.9, 3594 (2000)

38. Ghosh, S., Bharuthram, R.: Ion acoustic solitons and double layers in electron-positron-ion plasmas with dust particulates. Astrophys. Space Sci. 314, 121 (2008)

39. Dutta, D., Singha, P., Sahu, B.: Interlaced linear-nonlinear wave propagation in a warm multicomponent plasma. Phys. Plasmas 21, 122308 (2014)

40. Khaled, M.A.H.: Dust ion acoustic solitary waves and their multidimensional instability in a weakly relativistic adiabatic magnetized dusty plasma with two different types of adiabatic electrons. Ind. J. Phys. 88, 647 (2014)

41. Shahmansouri, M., Shahmansouri, B., Darabi, D.: Ion acoustic solitary waves in nonplanar plasma with two-temperature kappa distributed electrons. Ind. J. Phys. 87, 711 (2013)

42. Hussain, S., Akhtar, N.: Korteweg de Vries Burgers equation in multi-ion and pair-ion plasmas with Lorentzian electrons. Phys. Plasmas 20, 012305 (2013)
43. Malfliet, W.: Solitary wave solutions of nonlinear wave equations. Am. J. Phys. 60(7), 650 (1992)

44. Malfliet, W.: The tanh method: a tool for solving certain classes of nonlinear evolution and wave equations. J. Comput. Appl. Math. 164, 529 (2004)

45. Brinca, A.L., Tsurutani, B.T.: Unusual characteristics of the electromagnetic waves excited by cometary new born ions with large perpendicular energies. Astron. Astrophys. 187, 311 (1987)

46. Han, J.N., Duan, W.S., Li, J.X., He, Y.L., Luo, J.H., Nan, Y.G., Han, Z.H., Dong, G.X.: Study of nonlinear electron-acoustic solitary and shock waves in a dissipative, nonplanar space plasma with superthermal hot electrons. Phys. Plasmas 21, 012102 (2014)

47. Masood, W., Karim., Shah, H.A., Siddiq, M.: Drift ion acoustic shock waves in an inhomogeneous two-dimensional quantum magnetoplasma. Phys. Plasmas. 16, 042108 (2009)

48. Sabetkar, A., Dorranian, D.: Role of superthermality on dust acoustic structures in the frame of a modified Zakharov-Kuznetsov equation in magnetized dusty plasma. Phys. Scr. 90, 035603 (2015)

49. Sabetkar, A., Dorranian, D.: Parametric study of a Schamel equation for low-frequency dust acoustic waves in dusty electronegative plasmas. Phys. Plasmas 22, 083705 (2015) 